\title{
Ideologische Öffentlichkeit: Zur Organisation gesellschaftlicher Erfahrung im Kapitalismus
}

\author{
Sebastian Sevignani
}

Keywords: Erfahrung, Ideologie, Ideologietheorie, Hegemonie, Öffentlichkeit, Selbstvergesellschaftung, Handlungsfähigkeit, Kapitalismus

\section{Abstract}

Gesellschaftliche Erfahrung wird in einen aufsteigenden und einen absteigenden Kommunikationsprozess zwischen einfachen, mittleren und komplexen Teilöffentlichkeiten organisiert, der hier als ,ideologische Öffentlichkeit' bezeichnet wird. Deren Akteure sind eine Vielzahl antagonistischer Medien, deren unterschiedliche Positionen in den Massenmedien gemäß ihren unterschiedliche Machtressourcen verdichtet repräsentiert sind. Zudem gehören zur ideologischen Öffentlichkeit kompromissbildende Werte, wie etwa Unparteilichkeit, herrschaftsfreier Diskurs, Presseethiken, die eine überwölbende „bürgerliche Öffentlichkeit" bilden. Deren Repräsentation nehmen die Massenmedien einerseits für sich in Anspruch; sie kann aber auch von den antagonistischen Medien der unteren Ebenen der Öffentlichkeit an ihnen vorbei in Anspruch genommen werden. Der Effekt dieser komplexen Anlage ideologischer Öffentlichkeit ist es, ein Gemeinwesen zu behaupten, wo dieses angesichts antagonistischer Sozialbeziehungen nicht existiert und so zur Reproduktion gesellschaftlicher Antagonismen beizutragen. Die emanzipatorische Form von Öffentlichkeit bestimmt sich dann als "anti-ideologisch" und ist einem Erfahrungswachstum verpflichtet.

Sevignani, Sebastian. 2019. „Ideologische Öffentlichkeit. Zur Organisation gesellschaftlicher Erfahrung im Kapitalismus". In Ideologie, Kritik, Öffentlichkeit. Verhandlungen des Netzwerks Kritische Kommunikationswissenschaft, herausgegeben von Uwe Krüger und Sebastian Sevignani, 12-45. Universität Leipzig. DOI: 10.36730/ideologiekritik.2019.2

Sebastian Sevignani | Friedrich-Schiller-Universität Jena | sebastian.sevignani@uni-jena.de 


\section{Einführung}

Öffentlichkeit wird hier zunächst sehr weit als Organisierung von Erfahrungen gefasst (vgl. Negt und Kluge 1972). Dies hat zwei Vorteile. Erstens kann der Öffentlichkeitsbegriff (zunächst) deskriptiv verwendet werden und wird nicht sogleich als ein normativer Begriff gebraucht, wie er in vielen Demokratietheorien und kritischen Theorien zur Anwendung kommt (zum Überblick Ritzi 2013). Es ist also zunächst die Frage nach dem "Gebrauchswert" von Öffentlichkeit zu stellen. Gebrauchswert besitzt Öffentlichkeit, insoweit in ihr gesellschaftliche Erfahrung organisiert wird (Negt und Kluge 1972, 20; vgl. Klaus und Wischermann 2008, 106). Zweitens eignet sich diese Definition meines Erachtens gut, um das Öffentlichkeitsthema mit Ideologietheorie in Verbindung zu bringen. Dann verändert sich die Perspektive, nicht Öffentlichkeit selbst ist normativer Bezugspunkt kritischer Theorie, wie etwa prominent bei Habermas (1990, vgl. auch Fraser 2001), sondern die Art und Weise, wie Erfahrung organisiert wird, kommt in den Blick und dies kann ideologisch, d. h. kritikwürdig sein. Die Art und Weise, wie der öffentliche Kommunikationsprozess in einer Gesellschaft organisiert wird, gibt Hinweise darauf, so die diesem Beitrag zugrundeliegende Vermutung, wieso und wie sich auf Herrschafts- und Machtverhältnisse gestützte Ungleichheiten zwischen den Menschen reproduzieren.

Mit meinem Beitrag möchte ich zu den theoretischen Grundlagen einer kritischen Medien- und Kommunikationsforschung beitragen, wie sie bereits Anfang der 70er Jahre im deutschen Sprachraum begonnen ${ }^{1}$, dann aber hierzulande bis auf wenige Ausnahmen ${ }^{2}$ aufgegeben wurde und heute vor allem international weiterentwickelt wird ${ }^{3}$. Ein zentrales Anliegen dieser Forschungsrichtung ist es, die Gegenstandsbereiche Medien, Kommunikation und Öffentlichkeit in einem breiteren kapitalis-

$1 \quad$ vgl. insbesondere die versammelten Arbeiten in Prokop 1972; 1973; 1977 und die Arbeiten von Holzer 1973; 1994, die sich aus unterschiedlichen Quellen speisen, insbesondere der Kritischen Theorie der Frankfurter Schule (Adorno/Horkheimer), der Kritik der Politischen Ökonomie (im Anschluss an Marx) und einer medien- und kommunikationstheoretischen ergänzten historisch-materialistischen Sozialtheorie

$2 \quad$ vgl. insbesondere die Arbeiten von Manfred Knoche (programmatisch 1999)

3 vgl. insbesondere die Arbeiten von Christian Fuchs 2016; 2017; 2018; 2019 und meine eigenen Beiträge zur kritischen Medien- und Kommunikationsforschung: Sevignani 2016; 2018; 2019 
mustheoretischen Rahmen zu analysieren und Schlüsselkonzepte kritischer Gesellschaftstheorie (z. B. Frankfurter Schule, Cultural Studies, Politische Ökonomie, Sozialphilosophie, (Sozial-)Psychologie) für die kommunikationswissenschaftliche und mediensoziologische Forschung fruchtbar zu machen und dabei weiterzuentwickeln.

Im Folgenden konturiere ich ein Verständnis ideologischer Öffentlichkeit und greife dazu auf pragmatistische (Dewey), hegemonietheoretische (Gramsci, Hall), ideologietheoretische (Projekt Ideologietheorie, Haug), kritisch-psychologische („Holzkamp-Schule“) Theorien sowie die Kritische Theorie (Negt und Kluge) zurück. Einleitend plädiere ich für ein Zusammendenken von Ideologiekritik und Ideologietheorie (1), dann beschreibe ich mit Dewey den Zusammenhang zwischen Öffentlichkeit und Erfahrung (2), der anschließend kapitalismustheoretisch (3) und mit Gramscis Hegemonietheorie um seine konflikthaften Momente ergänzt wird (4). So informiert nutze ich ideologietheoretische Einsichten (5), um die Funktionsweise von Öffentlichkeiten in Gesellschaften mit ausgeprägter Zivilgesellschaft zu charakterisieren (6). Abschließend argumentiere ich für die konstitutive Rolle von Öffentlichkeiten in jedem Vergesellschaftungsprozess (7) und diskutiere die normative Perspektive der Selbstvergesellschaftung öffentlichkeitstheoretisch unter Rückgriff auf die verwendeten Theorietraditionen (8).

\section{Zum Verhältnis von Ideologiekritik und Ideologietheorie}

Ideologie ist ein Schlüsselbegriff kritischer Gesellschaftstheorie (vgl. Eagleton 2000; Rehmann 2008; Ritsert 2002; Žižek 1995). Zudem bildet er eine wichtige Brücke zwischen kommunikationswissenschaftlicher und mediensoziologischer Forschung zu einer interdisziplinären Gesellschaftstheorie (vgl. Downey, Titley und Toynbee 2014). Er trägt einen Großteil der Begründungslast, warum grundlegende gesellschaftliche Veränderung angesichts als problematisch identifizierter bestehender Verhältnisse ausbleiben, und zwar unter Bedingungen, wo sie nicht mit Zwang und Gewalt, sondern vermittelt über das eigene Wollen, Fühlen und Denken der beherrschten Subjekte aufrechterhalten werden. 
Bestimmte Ideen, Bedeutungsangebote und Denkformen sind dann funktionell, moralisch oder ethisch problematisch und kritikwürdig, weil sie in ihren Konsequenzen oder schon aufgrund ihrer Verfasstheit eine nichtnachhaltigen und inhärent krisengeschüttelte Gesellschaftsform, Ungleichheit (z. B. durch Ausbeutung, Unterordnung, Exklusion) oder Freiheitsverlust (z. B. durch Unterdrückung, aber auch Entfremdung, d. h. das Nicht-Verfügen-Können über die selbst mitverursachten Bedingungen des eigenen Lebens) legitimieren und aufrechterhalten. Der Ideologiebegriff wird hier "kritisch" verwendet und von einer neutralen Verwendungsweise abgegrenzt, wonach jegliche Weltanschauung oder die prinzipielle Standortgebundenheit und Historizität des Denkens schon als ideologisch bezeichnet wird.

Zwei Arten der Anwendung des Ideologiebegriffs können in der kritischen Forschung unterschieden werden. Die Ideologiekritiker*innen (vgl. Reitz 2004) fragen tendenziell nach dem "Was“ der Ideologie, d. h. nach ihrer konkreten Bedeutung und dem Inhalt verbreiteter Ideen; sie erforscht und kritisiert ein ideologisches Bewusstsein. Ihr Gegenstand ist (philosophisch gesprochen) der Begründungs- und Rechtfertigungszusammenhang von Ideologien. Ideologiekritik gibt Kriterien an, wonach sich ein ,wahres' von einem ,falschen' Bewusstsein unterscheiden lässt. Ist z. B. das verbreitete Deutungsangebot, wonach, wenn es „der Wirtschaft gut geht, es uns allen gut geht" in diesem Sinn ideologisch bzw. falsch? Die Ideologietheorie hingegen beleuchtet das "Wie“ der Ideologie, d. h. sie erforscht die Konstitutionsbedingungen von verbreiteten Bedeutungen, Inhalten und Bewusstseinsstrukturen (vgl. Rehmann 2008). Sie beschäftigt sich (wiederum philosophisch gesprochen) mit ihrem Entdeckungs- bzw. Entstehungszusammenhang.

Mit Hilfe der "Camera Obscura"-Metapher aus der Deutschen Ideologie von Marx und Engels $(1845-46 / 1969,26)$ kann dieser Unterschied von Ideologiekritik und Ideologietheorie verdeutlicht werden (vgl. Haug 1984). Ein Gegenstand wird hier innerhalb der Kamera seitenverkehrt und auf dem Kopfstehend projiziert (vgl. Abb. 1). Von ideologietheoretischem Interesse sind nicht in erster Linie der Gegenstand und seine Projektion, wie bei der Ideologiekritik, sondern die Funktionsweise der Kamera, die Projektionen verkehrt darstellt. Während konkrete Abbilder bzw. Ideologien historisch sehr variabel und zudem selbst dynamisch sind, ist die Anlage (der Kamera) bzw. die in den gesellschaftlichen Strukturen eingelassene Form des Ideologischen stabiler. Betrachtet man die Öffentlichkeits- 
struktur selbst als ideologisches Arrangement, dann wird einerseits deutlich, dass selbst öffentlich geäußerte Ideologiekritik sehr wahrscheinlich in ideologischen Formen stattfinden muss.

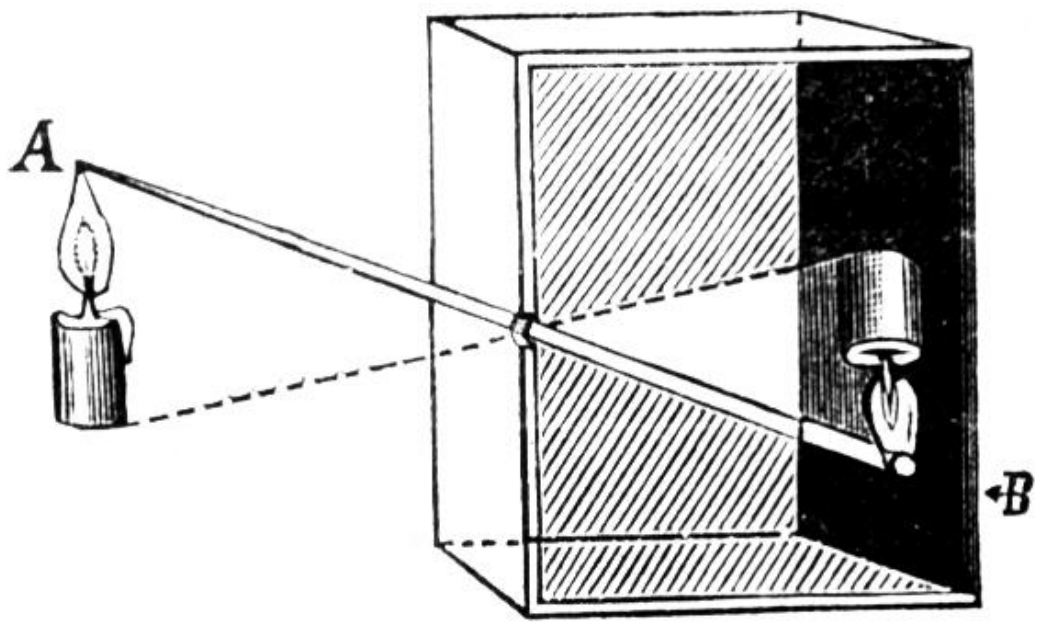

Abb. 1: Aufbau einer camera obscura (Quelle: https://commons.wikimedia.org/wiki/File:Camera_obscura_1.jpg)

Für die Ideologietheorie stellt sich allerdings ein Abgrenzungsproblem (vgl. Hall 1989, 186f.). Ein Rückzug ausschließlich auf das Problem des Entdeckungs- und Entstehungszusammenhangs kann nicht mehr angeben, warum es sich um einen kritischen und nicht um einen neutralen Ideologiebegriff handelt. Der ideologietheoretische Fokus auf das "Wie“ der Sicherung von Herrschaftsverhältnissen kommt also nicht daran vorbei auszuweisen, „was" das Herrschaftsverhältnis ausmacht und wie es sich in der Ideologie zeigt. Es geht also letztlich um die gelungene Verbindung von Ideologietheorie und Ideologiekritik (vgl. Koivisto und Pietilä 1996). Ideologische Öffentlichkeiten verweisen demnach auf eine spezifische Art, wie Erfahrung gesellschaftlich organisiert wird, die Herrschaftsverhältnisse absichert und deshalb kritisiert werden sollte. 


\section{3 Öffentlichkeit und Erfahrung}

Ebenso, wie es mir zunächst um einen möglichen deskriptiven Öffentlichkeitsbegriff geht, kann auch der Erfahrungsbegriff in dieser Weise verwendet werden 4 . Dass Menschen Erfahrungen machen, kann vorausgesetzt werden, dann stellt sich aber die Frage, wie dies genau funktioniert. Bei dem amerikanischen pragmatistischen Philosophen John Dewey findet sich eine ausgearbeitete Theorie der Erfahrung, die sich zwar normativ aufladen lässt (vgl. Jörke 2003; 2007), aber sich zunächst auch für deskriptive Zwecke gebrauchen lässt (Götz 2017, 12).

Deweys Ansatz wendet sich gegen eine Auftrennung von Theorie und Praxis in der Wissenschaft und auch der Politik; er geht davon aus, dass in der Praxis gewonnene Erfahrungen im Vergleich zur Reflexion und Erkenntnis einen primären Status besitzen. Ausgangspunkt ist eine präreflexive Weltbeziehung des Subjekts, das in einen Strom der Erfahrung gestellt ist und aufgrund gemachter Erfahrungen relativ unproblematisch und gemäß seinen Bedürfnissen mit Dingen und anderen Menschen umgehen kann. Es gibt bei ihm keinen Nullpunkt und keinen plötzlichen Einbruch der Erfahrung, sondern jede Erfahrung wird vor dem Hintergrund von bereits Erfahrenem, von habits, gemacht. Dewey betrachtet immer eine Sequenz von Erfahrung, ausgehend von Erfahrenem wird die zukünftige Erfahrung konturiert. Dies kann das Prinzip der Kontinuität genannt werden (vgl. Dewey 1986, 285).

Erfahrung ist zunächst gekennzeichnet von passiven Elementen des Erleidens und aktiven Elementen der Bearbeitung. In dieser primären Erfahrungssituation besteht eine relative Einheit von Subjekt und Objekt. Erst bei einer Irritation, wenn im Erfahrungsstrom ein Problem auftritt und die beschriebene Anpassung an die Welt prekär wird und Handlungsunsicherheit eintritt, kommt es zu einer sekundären Form der Erfahrung, bei der einzelne Aspekte aus dem Erfahrungsstrom selektiv hervorgehoben werden. Subjekt und Objekt treten dann auseinander und eine

$4 \quad$ Negt und Kluge konzipieren den Zusammenhang von Öffentlichkeit und Erfahrung so, dass Öffentlichkeiten Erfahrungen blockieren (bürgerliche Öffentlichkeit, Produktionsöffentlichkeit) oder ermöglichen (proletarische Öffentlichkeit) können. Erfahrung fungiert hier als normativ gehaltvoller, aber meinem Eindruck nach gleichsam unterbestimmter Begriff, von dem aus Öffentlichkeiten kritisiert werden können. Deshalb wende ich mich zunächst einer anderen Theorie der Erfahrung zu. 
Reflexion auf in die Krise geratene Umgangsweisen, Handlungsmuster und die in ihnen immer schon eingelassenen Normen und Werte setzt ein. Es kommt zu einer reflexiven Situationsdeutung und der Erarbeitung von Problemlösungsstrategien zur (Wieder-)Erlangung von Handlungsfähigkeit. Erfahrung kann dann sowohl in der (sozialen) Natur (primäre Erfahrung), also auch von ihr gemacht werden (sekundäre Erfahrung).

Der Zusammenhang zwischen Erfahrung und Öffentlichkeit ist laut Dewey der folgende: Eine Öffentlichkeit wird von denjenigen gebildet, deren private Handlungsfähigkeit prekär wird, weil sie von problematischen Folgen in einer bestimmten gesellschaftlichen Situation betroffen sind, und die dann versuchen ihre Handlungsfähigkeit zurück zu erlangen oder zu erhöhen (vgl. Dewey 1996, 20ff.). Öffentlichkeit ist ein sozialer Problemlösungsmechanismus und erlaubt die reflexive Distanzierung (sekundäre Erfahrung), die Verständigung über ein Problem und den Entwurf von Problemlösungsstrategien. Solche „schwachen“ Öffentlichkeiten werden zu "starken“ Öffentlichkeiten, wenn die Betroffenen zusätzlich reale Möglichkeiten der praktischen Umsetzung und Erprobung von Lösungsstrategien bekommen ${ }^{5}$. Nach dem Prinzip der Kontinuität präformieren vorangegangene Problemöffentlichkeiten sowohl die Situationsdeutung und die Entwicklung von Lösungsstrategien, als auch die Möglichkeiten ihrer Erprobung.

Eine häufig geäußerte Kritik an Dewey ist, dass seine Theorie die Konflikthaftigkeit von Erfahrungen unterschätzt und deshalb für eine kritische Theorie ergänzt werden muss:

- Konflikte im individuellen Erfahrungsprozess bleiben weitgehend ausgeblendet. Bei Dewey scheint es manchmal so, als ginge der Erfahrungsprozess der Probleme und ihrer Folgen der Öffentlichkeitsbildung einfach voraus (vgl. Götz 2017, 42). Dass subjektive Erfahrungen aber selbst durch soziale Konflikte und mithin ideologische Öffentlichkeiten beeinflusst sind, kann trotz der Annahme des Prinzips der Kontinuität mit pragmatistischen Mitteln nur unzureichend aufgeklärt werden, weil die pragmatische Theorie mit dem Problem einsetzt und dessen Zustandekommen unterbelichtet lässt. 
- Bei Dewey erfährt man wenig darüber, wie gerade die kapitalistische Gesellschaft Probleme erzeugt.

- Während Dewey Konflikte nur zwischen alten, etablierten Problemlösungen repräsentierenden Öffentlichkeiten und neuen Problemöffentlichkeiten situiert (vgl. Götz 2017, 32f.), nimmt er innerhalb von Öffentlichkeiten aufgrund des geteilten konstitutiven Problembezugs relativ homogene Interessen an.

\section{Erfahrung und kapitalistische Gesellschaftsstruktur}

Die aufgeworfenen Probleme fehlender Konflikthaftigkeit in der pragmatistischen Öffentlichkeitstheorie können sinnvoll unter Rückgriff auf alternative kritische Theorien bearbeitet werden. Zunächst ist es in dieser Theorietradition eine verbreitete Annahme, dass gegenwärtige Gesellschaften zutreffend als kapitalistische beschrieben werden können. Dies bedeutet $\mathrm{u}$. a., dass in den sozialen Verhältnissen Mechanismen liegen, die das Glück der Starken mit dem Leid der Schwachen verbinden (vgl. Boltanski und Chiapello 2006, 398). Kapitalistische Gesellschaften sind also Klassengesellschaften, die sich z. B. durch die Mechanismen von Ausbeutung, Enteignung und Klassifizierung ökonomisch, aber auch politisch und kulturell als solche reproduzieren. ${ }^{6}$ Unterschiedliche Klassen sind zwar aufeinander angewiesen, aber durch ihre Beziehung zueinander gleichzeitig in eine ungleiche vertikale Schichtung gebracht. Es ist leicht ersichtlich, dass in der Struktur so einer Gesellschaft ein wichtiger Ursprung für Konflikte, die Erfahrung von Problemen und damit für die Bildung von Öffentlichkeiten nach Dewey liegt. Das invers-interdependente Verhältnis des Glücks der Starken mit dem Leid der Schwachen produziert immer wieder Ausgangslagen für Probleme, die habituell nicht gelöst werden können.

Soziale Probleme ergeben sich laufend aufgrund dieser antagonistischen Verfasstheit kapitalistischer Gesellschaften. Dabei ist es typisch für den Kapitalismus, dass sich ökonomische Klassenwidersprüche mit Antagonismen entlang weiterer Achsen, wie ,race' und Geschlecht überschneiden. Mit Stuart Hall und Nancy Fraser, die eine nichtreduktionistische, ,intersektionale' kritische Theorie des Kapitalismus konzipieren, bin ich der Meinung, dass sich diese Widersprüche jeweils nur historisch spezifisch, $\mathrm{d}$. $\mathrm{h}$. in vom Kapitalismus geprägten Verhältnissen ausdrücken können (vgl. Fraser und Jaeggi 2018; Hall 1994; Demirović 2018). 
Aus dieser Perspektive einer strukturell argumentierenden Kapitalismusund Klassentheorie stellt sich aber empirisch sofort ein doppeltes Problem. Grundsätzlich gleiche gesellschaftliche Verhältnisse (zum Beispiel Benachteiligungen, Unterdrückungen) werden von verschiedenen Individuen unterschiedlich erfahren und grundsätzlich gleiche Erfahrungen werden von verschiedenen Individuen, insbesondere aufgrund unterschiedlicher Interessen, unterschiedlich „verarbeitet" werden, d. h. führen dann auch zu unterschiedlichen Handlungen (Alltagshandeln, politisches Engagement, Wahlen).

Dieser Erfahrungsprozess (Erfahrung machen und die davon nicht zu trennende ,Verarbeitung') wird durch Öffentlichkeit organisiert. Gesellschaftliche Bedingungen, zu denen neben der Klassenlage, auch die ideologische Organisation von Erfahrungen, sowie einzelne Ideologien gehören, legen spezifische Bedeutungen für die Subjekte nahe, ohne sie aber zu determinieren.

D. h. der Zusammenhang zwischen gesellschaftlichen Bedingungen und subjektiver Bedeutung muss noch weiter expliziert werden. Ich komme darauf und auf den Interpretationsvorschlag, den die Kritische Psychologie in diesem Zusammenhang macht, sowie auf die öffentlichkeitstheoretische Perspektive, die ich damit verbinde, zurück (vgl. Abschnitt 7 und 8 ).

\section{$5 \quad$ Hegemonietheoretische Erweiterung des Erfahrungsbegriffs}

Auch Antonio Gramscis Hegemonietheorie setzt bei der (Klassen-)Struktur der Gesellschaft an, denn „diese grundlegende Anordnung ermöglicht zu untersuchen, ob in der Gesellschaft die notwendigen und hinreichenden Bedingungen für ihre Umgestaltung vorhanden sind, ermöglicht also, den Grad an Realismus und Umsetzbarkeit der verschiedenen Ideologien $\mathrm{zu}$ kontrollieren, die auf ihrem eigenen Boden entstanden sind, dem Boden der Widersprüche, die sie bei ihrer Entwicklung hervorgebracht hat" (Gramsci 1991ff., zit. nach Becker et al. 2013, 31). Klassenlagen drücken sich politisch aus, wenn sie einen gewissen Grad , an Homogenität, Selbstbewusstsein und Organisation“ (Gramsci 1991ff., zit. nach Becker et al. 2013,31 ) erreichen. Durch die Betonung des Organisations-Aspekts wird Gramscis Klassentheorie eine öffentlichkeitssensible Klassentheorie. 
Gramsci zeigt Stufen auf, die ausgehend von der sozio-strukturellen Lage einer Gruppe genommen werden müssen, um gesellschaftliche Macht zu erringen. Er unterscheidet zwischen einer kooperativ-ökonomischen Stufe, auf der aus den gemeinsamen Problemen, die sich mit der jeweiligen Stellung einer Gruppe im Produktionsprozess ergeben, ein Gefühl der Gemeinsamkeit entsteht. Auf der klassen-korporatistischen Stufe wird aus der gemeinsamen Problemlage eine Interessensolidarität entwickelt, die sich „aber noch auf bloß ökonomischem Gebiet" (ebd.) abspielt. Interessen werden innerhalb eines bestehenden Problemrahmens vertreten. Auf der politisch-hegemonialen Stufe wird dieser korporative Umkreis überschritten. Kennzeichen ist das Erringen „kultureller Führung“, d. h. die eigenen Interessen müssen $\mathrm{zu}$ Interessen anderer untergeordneter Gruppen werden (ebd.). So können bestehende Machtverhältnisse herausgefordert werden, wobei die so entstandene politische Klasse „über die Einheitlichkeit der ökonomischen und politischen Ziele hinaus auch die intellektuelle und moralische Einheit bewirkt, alle Fragen, um die der Kampf entbrannt ist, nicht auf die korporative, sondern auf eine ,universale' Ebene stellt und so die Hegemonie einer grundlegenden gesellschaftlichen Gruppe über eine Reihe untergeordneter Gruppen herstellt" (ebd., 32). In dieser hegemonialen Phase müssen fortwährend Interessengleichgewichte hergestellt werden, „Gleichgewichte, in denen die Interessen der grundlegenden Gruppe überwiegen, aber nur bis zu einem gewissen Punkt, also nicht bis zum nackten korporativ-ökonomischen Interesse" (Gramsci 1991ff., zit. nach Becker et al. 2013, 32).

Hegemoniebildung ist in Gesellschaften mit ausgeprägter Zivilgesellschaft ein entscheidendes Moment, um ein stabiles ,Integral' der Herrschaft zu errichten, das unterschiedliche Gesellschaftsbereiche wie die Ökonomie, den Staat mit seinen Exekutivorganen und die zivilgesellschaftliche Kultur in ein Herrschaftsprojekt einspannt. ${ }^{7}$ Eine in einer spezifischen geschichtlichen Zeit geglückte Artikulation dieser Bereiche nennt Gramsci "historischen Block". Mit dem Begriff der Hegemonie macht Gramsci darauf aufmerksam, dass Herrschaftsverhältnisse durch politisch-kulturelle Führung abgesichert werden müssen und nicht auf

vgl. zu einer Historisierung des Hegemoniebegriffs (Martin und Wissel 2015, 222): „Die politischen, kulturellen und ökonomischen Dimensionen von Hegemonie können in unterschiedlichem Grad, in unterschiedlicher Zusammensetzung und im Hinblick auf gesellschaftliche Gruppen, Klassen und Klassenfraktionen in unterschiedlichem Umfang realisiert sein in einer konkreten Gesellschaftsform bzw. Regulationsweise" . 
reiner (Staats-)Gewalt beruhen können. In der Zivilgesellschaft wird in Medien, Bildungsinstitutionen etc. um Konsens gerungen, „all das, was die öffentliche Meinung direkt oder indirekt beeinflußt oder beeinflussen kann, gehört zu ihr" (Gramsci 1991ff., 374) und „die sogenannte ,öffentliche Meinung' ist eng mit der politischen Hegemonie verknüpft. Sie ist Berührungspunkt zwischen ,Gesellschaft' und ,Staat', zwischen Konsensus und Macht" (Gramsci 1991ff., 916f.).

Die Rolle der Intellektuellen ist in der Herausbildung von Hegemonie entscheidend. Für Gramsci gibt es keine Organisation und Interessenverallgemeinerung ohne Intellektuelle (vgl. Demirović und Jehle 2005: 1270). Bei intellektueller Tätigkeit geht es „nicht nur um Konzipierung neuer Gedanken, sondern auch um ihre ,Vergesellschaftung' und Durchsetzung, mithin die Fähigkeit, einen neuen Alltagsverstand zu prägen" (Demirović und Jehle 2005: 1269). Die ,Organizität' eines Intellektuellen bestimmt sich für Gramsci am Grad seiner Verbundenheit mit einer gesellschaftlichen Gruppe. Da es keinen Nullpunkt der Hegemonie gibt, sondern hegemoniale Strategien immer in bestehenden hegemonial geprägten Strukturen agieren, finden die organischen Intellektuellen bereits "traditionelle Intellektuelle“ vor, die die erfolgreichen Gruppen früherer Hegemoniekämpfe repräsentieren, sich aber aus ihrer organischen Verbindung zu diesen gelöst haben und in relativer Autonomie in den zivilgesellschaftlichen Institutionen agieren. Neben den organischen (direkter Bezug zur Lebensweise einer Klasse) und den traditionellen Intellektuellen (in den Institutionen der Zivilgesellschaft) kann auch zwischen ,kleinen' und ,großen' Intellektuelle unterschieden werden (vgl. Demirović 1999, 26). Vom kleinen zum großen Intellektuellen nimmt der Grad an Konsensausarbeitung durch Aufnahme, Verfolgung, Erklärung von Differenzen zu Lasten der Konkretheit der Interessenvertretung zu. Große Intellektuelle finden sich in der Philosophie, der Kunst und den Wissenschaften; kleine Intellektuelle sind Verwalter*innen und Populisator*innen bereits bestehender intellektueller Kategorien. Hegemoniale Strategien (organischer Intellektueller) können sich dann nicht nur auf die tatsächliche Verallgemeinerung eigener und Einbindung anderer Interessen richten, sie können auch darauf zielen, andere Gruppen gewissermaßen intellektuell zu ,köpfen', d. h. deren organische Intellektuelle für sich zu gewinnen.

Die antagonistische (Klassen-)Gesellschaft, die sich im Modus der Hegemonie reproduziert, wirkt in die subjektive Organisation von Erfah- 
rung hinein. Gramsci bietet hier mit der Kategorie des „Alltagsverstandes“ einen Anknüpfungspunkt, wie der Erfahrungsprozess und das Deweysche Prinzip der Kontinuität in einer hegemonial-herrschaftlich strukturierten Gesellschaft verstanden werden kann und zwar einen der, wie gezeigt werden soll, auch mit Öffentlichkeiten, d. h. der intersubjektiven Organisation von Erfahrung in Verbindung steht. Die zwei wichtigen Punkte, auf die Gramsci mit der Konzeption des Alltagsverstandes im Vergleich mit Dewey hinweist, sind, dass der Übergang von primären zu sekundären Erfahrungen nicht selbstverständlich ist, Öffentlichkeiten also möglicherweise gar nicht gebildet werden, und dass hierbei und generell in jedem Erfahrungsprozess gesellschaftliche Herrschafts- und Machtverhältnisse immer bereits wirken.

Der Alltagsverstand organisiert den primären Erfahrungsprozess. Er erscheint als bewährter, erfahrungsgesättigter und gesicherter Bestand an Wissens-, Denk- und Gefühlsformen und besteht aus populärem Wissen, spontaner Philosophie, Vorurteilen, Werten und Moralvorstellungen. Ganz im Sinne des Prinzips der Kontinuität sind dies sedimentierte Erfahrungen, die den Hintergrund bilden, vor dem neue Erfahrungen gemacht werden können. Die Elemente des Alltagsverstandes speisen sich aus unterschiedlichen Quellen, wie der Vergangenheit (z. B. aus tradierten Überzeugungen und in der eigenen sozialen Laufbahn gesammelten Erfahrungen), aus Muster von Lebensformen und -vorstellungen, die sich aus der Zugehörigkeit zu bestimmten Milieus oder unterschiedlichen Rollenerwartungen ergeben sowie aus Problemen und Erfahrungen, die sich aus konkreten Handlungskontexten, wie z. B. der Familie oder dem Betrieb ergeben. Zudem wirken gesellschaftlich verbreitete Deutungsangebote und die wahrgenommene öffentliche Meinung auf ihn ein. Der Begriff des Alltagsverstandes verbindet die gesellschaftliche (Makro-)Ebene und die dort zirkulierenden Ideologien mit einem Verständnis von Ideologie "gelebter, habitueller gesellschaftlicher Praxis“ (Eagleton 2000, 136) auf der subjektiven (Mikro-)Ebene.

Bei genauerer Betrachtung, so Gramsci, erweist sich dieser Bestand an unterschiedlichen Elementen als ein fragmentiertes und widersprüchliches Terrain, auf dem verschiedene gesellschaftliche Gruppen um subjektive Zustimmung ringen. Der Kampf um Zustimmung oder „kulturelle Führung" erfolgt über die (Neu-)Zusammensetzung und vorübergehende Strukturierung und Hierarchisierung der Elemente des Alltagsverstandes. Stuart Hall argumentiert dementsprechend, dass „keine Strategie 
[...] so erfolgreich [ist] Konsens herzustellen, wie diejenige, der es gelingt, sich in den widersprüchlichen Elementen des Alltagsverstandes, des Lebens und Bewusstseins der Bevölkerung zu verwurzeln“ (Hall 2014c, 236).

Bestimmte gesellschaftliche Deutungen erscheinen insofern plausibel und subjektiv zustimmungsfähig, wenn sie

- an Elemente aus dem Alltagsverstand anknüpfen können,

- mehrere Elemente in eine ,stimmige' Ordnung zu bringen vermögen, also z. B. Probleme im Betrieb mit tradiertem Handlungswissen und familiären Werten in Übereinstimmung bringen, und

- es gelingt nicht passförmige Elemente zu desartikulieren und in ihrer Deutungsfähigkeit herabzusetzen.

Mit Gramsci kann Deweys Erfahrungstheorie dahingehend erweitert werden, dass erstens deutlich wird, wie zunächst die subjektive Situationsdeutung und Problemdefinition und dann auch die intersubjektive Entwicklung von Lösungsstrategien sowie die Bewertung der Möglichkeiten ihrer Erprobung vom bereits hegemonial geprägten Alltagsverstand der Einzelnen und der Betroffenengruppen beeinflusst sind. Zweitens ist der Übergang von primären zu sekundären Erfahrungen nicht selbstverständlich. Gramsci weist darauf hin, dass die widersprüchlichen Elemente im fragmentierten Alltagsverstand, die dementsprechend konfligierende Situationsdeutungen nahelegen, sich passivierend auswirken können (Gramsci 1991ff., 1384). Fragmente des Alltagsverstandes kompensieren sich gegenseitig und verhindern eine Problematisierung und den Beginn von Reflexion, die erst eine Hierarchisierung und einen Ordnungsprozess des Alltagsverstandes in Gang bringen würde. Öffentlichkeiten bilden sich also möglicherweise gar nicht. Drittens macht Gramsci auf die Möglichkeit eines Widerspruchs zwischen primärer und sekundärer Erfahrung, zwischen praktischem und verbalem Bewusstsein, aufmerksam. Während das praktische Bewusstsein ein „realistisches“ und „materialistisches“ Element des „gesunden Verstandes“ enthält (Gramsci 1991ff., zit. nach Becker et al. 2013, 135, 138), was sich in der Erfahrung von kausalen Zusammenhängen in der sozialen Welt ausdrückt, die nicht zuletzt auch durch die Gesellschaftsstruktur und eigene Klassenlage gegeben sind, ist das verbale, öffentliche Bewusstsein demgegenüber vermittelnd-abstrakt und glättet möglicherweise Widersprüche der praktischen Ebene. So kann z. B. die Situation mit Vorgesetzten im Betrieb als 
ungleich und hierarchisch erfahren werden, während im verbalen Bewusstsein Ideen der Sozialpartnerschaft vorherrschend sind. Teile des Alltagsverstandes widersprechen hier der öffentlichen Meinung. Dieses materialistisch-realistische Element hat für Gramsci das Potenzial, hegemoniale Weltauffassungen oppositionell, subversiv oder abweichend zu deuten. In einer positiven Interpretation, die auf mögliche Ähnlichkeiten zwischen Dewey und Gramsci abzielt, wäre dieser Teil der Problemgenerator und der Auslöser für gegenhegemoniale Öffentlichkeitsbildung, in der die öffentliche Meinung diese Erfahrung auch wirklich kohärent ausdrückt (vgl. hierzu Abschnitt 9).

\section{Theorie des Ideologischen}

Mit Gramsci kann Erfahrung und ihre Organisation in Öffentlichkeiten als hegemonialer Prozess im Kampf um Machtpositionen verstanden werden. Diese hegemonietheoretischen Überlegungen beeinflussten stark die im Anschluss an die Arbeiten des Projekts Ideologietheorie (PIT 1978) entwickelte Theorie des Ideologischen (Haug 1993). Sie soll im Folgenden für die Öffentlichkeitstheorie nutzbar gemacht werden. Eingangs wurde auf die Notwendigkeit einer Verbindung von Ideologietheorie und Ideologiekritik hingewiesen; die Theorie des Ideologischen teilt diesen Anspruch und grenzt sich so von einer Hegemonietheorie ab, der es um die neutrale Analyse von Hegemonieprozessen geht. Ideologische Formen der Organisation von Erfahrung werden hier vor dem ethischen Hintergrund einer möglichen Selbstvergesellschaftung der Menschen sichtbar gemacht (vgl. Koivisto und Pietilä 1996). Damit geht eine Unterscheidung der Richtung der Vergesellschaftungsprozesse einher: Die entscheidende Frage ist dann, ob diese Prozesse "von unten" ausgehen und ob dies auch in den Resultaten der Vergesellschaftung sichtbar bleibt oder ob sie "von oben“, von den Eliten ausgehen.

Das Ideologische wird bestimmt als entfremdete ideelle Vergesellschaftung von oben (vgl. Haug 1993, 72f.). Was heißt das? Hier wird angenommen, dass die Menschen Fähigkeiten zur öffentlichen Selbstvergesellschaftung besitzen, also in der Lage sind, Probleme des Zusammenlebens über die Bildung von Öffentlichkeiten so zu lösen, dass ihre Handlungsfähigkeit gesichert oder gar erweitert wird. In einer für Klassengesellschaften typischen Situation strukturell antagonistischer Interessen 
werden diese Vergesellschaftungskompetenzen aber an „ideologische Mächte“, wie den Staat, das Recht und die (Massen-) Medien, abgetreten. Denn auf dieser ideologischen Ebene ist es möglich, Kompromisse zu bilden und so trotz einander antagonistisch gegenüberstehenden Interessen gemeinsam handlungsfähig zu bleiben. Die Auslagerung von Kompetenzen auf diese Mächte ist also subjektiv in der Sicherung von Handlungsfähigkeit begründet und wird deshalb von beiden Seiten des Antagonismus, den Herrschenden (die ihre Handlungsfähigkeit auf Kosten anderer ausweiten) und Beherrschten (die durch ein zumindest implizites, Bündnis mit den Herrschenden' auch handlungsfähig bleiben), gehalten.

So hat das Ideologische zugleich einen antagonistischen und einen allgemeinen Aspekt: Die allgemeine Bindungskraft entsteht durch eine bedeutungsoffene Form, die jeweils konkret gefüllt wird und deren Bedeutung die unterschiedlichen gesellschaftlichen Interessen repräsentiert. Das antagonistische Element in den ideologischen Formen besteht in einer Verdichtung von Kräfteverhältnissen analog der von Gramsci beschriebenen Hegemonialwerdung einer Klasse. Im Prozess der Kompromissbildung spiegeln sich die unterschiedlichen Kräfteverhältnisse, und mächtige Interessen definieren stärker das, was als Allgemeines gilt. Zusammenfassend wird diese hegemoniale Logik von Haug als „, antagonistische Reklamation des Gemeinwesens" (Haug 1993, 59ff., 84ff.) durch kulturellpolitische Äquivokation von Interessen gefasst. ${ }^{8}$

So bleiben jedoch im Effekt die antagonistische Struktur der Gesellschaft und herrschende Machtungleichheiten unangetastet bzw. ist die Kompromissbildung zwischen antagonistischen Interessen innerhalb dieser sich über der Basis der Gesellschaft konstituierenden Mächten die Bedingung für ihre Reproduktion: „Die ideologischen Apparate der bürgerlichen Gesellschaft organisieren das (Er-)Leben der Klassengesellschaft als Erleben der Klassenlosigkeit" (Haug 1993, 55). Im selben Moment kommt aber durch das Ideologische eine Vorahnung eines wirklichen Gemeinwesens, d. h. einer Gesellschaft ohne Antagonismus in die Welt und erhält gesellschaftlich Bedeutung. Dies ist das ideelle Moment der ideologischen Vergesellschaftung. Die bestehenden Verhältnisse werden zwar

$8 \quad$ Zur diskursiven Logik dieser Äquivokation siehe die Ausführungen von Laclau (2010; vgl. auch Marchart 2017), dessen Theorie jedoch - entgegen Gramsci, Hall und Haug die objektive Klassenstruktur in symbolische Diskurse auflöst (vgl. Opratko 2014, 148; Rehmann 2008, 136ff.; Hall 2014, 112f.) 
durch das "Allgemeine“ gestützt, welches aber - einmal in der Welt auch gegen sie und die ideologischen Apparate, Praxen und Akteure angerufen werden kann. Ideologische Formen spalten sich auf in universale Werte und institutionelle (Staats-)Apparate. Letztere geben vor, die universalen Werte zu repräsentieren, und legitimieren sich von dorther. Das Ideologische ist also einerseits durch einen „,weltlichen Himmel“ der Apparate der Kompromissbildung und Verdichtung von Interessen und andererseits durch eine „himmlische Welt" aus einer von den Kräfteverhältnissen abgelösten ideellen Vorwegnahme der Kompromissbildung in Gestalt von Begriffen und Werten mit allgemeiner Bindungskraft gekennzeichnet: „Es ist, als entspräche die innergesellschaftliche Spaltung dem Spalt in der ideologischen Macht, der ein Spalt ist zwischen ,übergesellschaftlichem' Apparat und den jenseitigen Mächten, denen er zu dienen beansprucht. (...) Das ,Oben' verdoppelt sich in einen weltlichen Himmel und eine himmlische Welt" (Haug 1993, 85).

Die materialisierten Verdichtungen antagonistischer Kräfteverhältnisse, also die ideologischen Apparate (des weltlichen Himmels), errichten Eintrittsbarrieren in Form von feldspezifischen und habituellen Logiken und schließen sich nach unten ab. Dieser Ausschluss legitimiert sich vor den symbolischen Verdichtungen der Kräfteverhältnisse, den allgemeinverbindlichen Werten (der himmlischen Welt).

Zwischen diesen ideologischen Werten und den ideologischen Apparaten können aber v. a. in Krisenzeiten Widersprüche oder Risse in ihrer Repräsentationsfunktion entstehen. Ideologische Werte können an den Apparaten vorbei von unten angerufen werden mit dem hegemonialen Ziel, Letztere umzuorganisieren, d. h. ihre Rekrutierungslogiken und Spielregeln zu ändern, aber auch um ihre Akteure auszutauschen.

Die Unterscheidung zwischen „unten“ (Gesellschaft) und „oben“ (ideologische Mächte), sowie der Bezug auf die Selbstvergesellschaftungskompetenzen der Menschen trägt das kritische Potenzial der Theorie des Ideologischen: "Ausgangspunkt und Fluchtpunkt der Analyse ist die Selbstvergesellschaftung der Menschen im Sinne einer gemeinschaftlich-konsensuellen Kontrolle der gesellschaftlichen Lebensbedingungen" (Haug 1993, 47), was gleichbedeutend mit der Aufhebung der Klassengesellschaft ist. Vor diesem Hintergrund kann Haug auch zwischen dem Ideologischen und dem Proto-Ideologischen unterscheiden. Letzteres ist die notwendige Abgabe von Vergesellschaftungskompetenzen in einer arbeitsteiligen und funktional-differenzierten modernen Gesellschaft an 
bestimmte Gruppen, wie z. B. die Wahl eines Rates, der für eine bestimmte Zeit die Geschicke eines Unternehmens führt. Der entscheidende Unterschied ist die Wirkungsweise dieser Führung: Wird sie von oben oder von unten ausgeführt? Ideologisch ist nur die Wirkungsweise von oben zu nennen (vgl. Abb. 2).

ideologische Werte („,himmlische Welt ${ }^{u}$ )

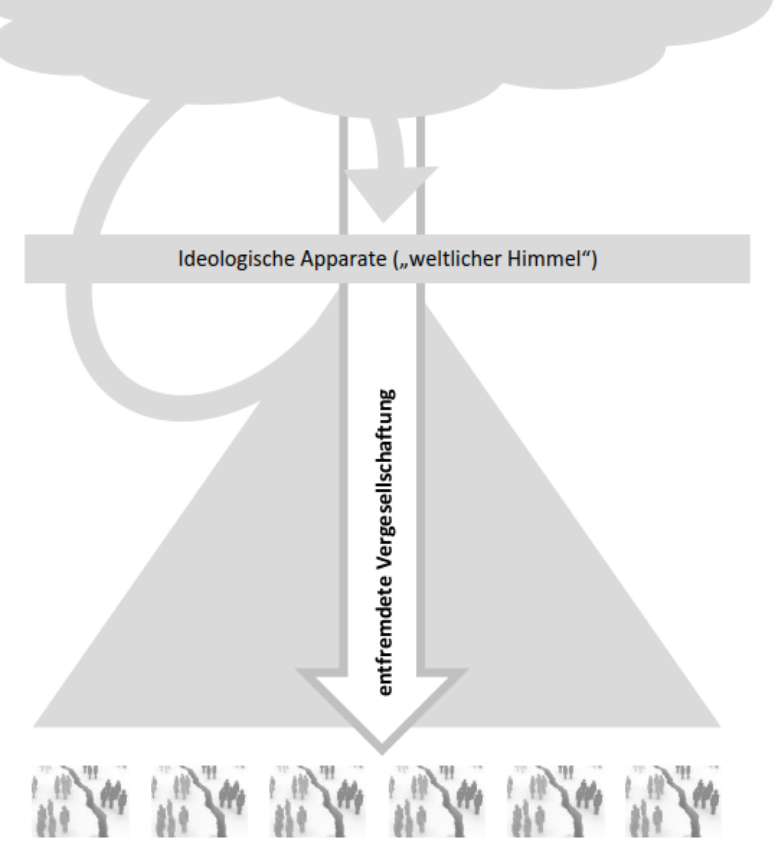

antagonistische Interessen und ungleiche Kräfteverhältnisse in der kapitalistischen Produktionsweise

\section{horizontale Selbstvergesellschaftung \\ kooperative Kontrolle der Lebensbedingungen}

Abb. 2: Schema der Theorie des Ideologischen (eigene Darstellung) 


\section{Ideologische Öffentlichkeiten}

Während Dewey zeigt, dass sich Öffentlichkeiten um die Erfahrung von Problemen bilden, und mit Gramscis Hegemonietheorie Öffentlichkeiten als Austragungsorte von Machtkämpfen um kulturelle Führung sichtbar werden, kann mit der Theorie des Ideologischen deutlich gemacht werden, dass die Organisation von Öffentlichkeiten selbst ideologisch sein kann. Entsprechend argumentiert auch Alex Demirović: „Hegemonie wird nicht allein in der Öffentlichkeit und um die Grenzen der Öffentlichkeit praktiziert, sondern Öffentlichkeit ihrerseits praktiziert Hegemonie, eine Form von kultureller Herrschaft, insofern mit einem enormen Form, Regel-, Anstands-, und Hierarchiebewußtsein die freie diskursive Praxis von den sozialen Akteuren getrennt, reduziert, kontrolliert, diszipliniert und normalisiert wird" (Demirović 1997, 182). Im Folgenden soll nun die Rolle von Öffentlichkeiten und Medien in dieser Theorie des Ideologischen bestimmt werden. Wie werden gesellschaftliche Erfahrungen medial-ideologisch organisiert?

Hilfreich ist hier zunächst das Mehrebenenmodell von Öffentlichkeit (vgl. Klaus und Wischermann 2008; Klaus und Drüecke 2017), da es sich einerseits wiederum um ein normativ sparsames, eher deskriptives Modell handelt und andererseits sowohl die Theorie des Ideologischen als auch Gramscis Hegemonieprozess eine topische Konzeption darstellen.

In einfachen Öffentlichkeiten der interpersonellen Kommunikation und unmittelbaren Begegnung sind die funktionalen Rollen zwischen Kommunikator*innen und Zuhörer*innen noch nicht differenziert und wechseln ständig. In mittleren Öffentlichkeiten bilden sich erste funktionale Rollen und Zuweisungen heraus, es gibt bedeutende Kommunikator*innen und es entstehen Kommunikationsregeln. Prototypische mittlere Öffentlichkeiten bilden sich um Vereine, Bürgerinitiativen und soziale Bewegungen. In komplexen Öffentlichkeiten sind Kommunikator*innen-Rollen weitgehend professionalisiert und die Beziehungen zu Medien und Teilöffentlichkeiten eingespielt, wobei das Publikum „immer abstrakter und in seinen Handlungsmöglichkeiten beschränkter" (Klaus und Wischermann 2008, 108) wird. Zwischen den Öffentlichkeitsebenen besteht ein komplexes Verhältnis, was nun mittels der Hegemonie- und Ideologietheorie besser verstanden werden kann. Als Verallgemeinerung aus den unzähligen Öffentlichkeiten konstituiert sich hegemonial ständig 
neu eine komplexe und aggregierte Öffentlichkeit, die ihrerseits die vielen Teilöffentlichkeiten rückstrukturiert. Es gibt also einen aufsteigenden und einen absteigenden Zusammenhang zwischen einfachen, mittleren und komplexen Öffentlichkeiten.

Ausgehend von den Problemerfahrungen kapitalistisch-antagonistischer Gesellschaften mit ihren sozialen Mechanismen der Ausbeutung, Unterordnung und Exklusion bilden sich zunächst einfache Öffentlichkeiten (kooperativ-ökonomische Ebene bei Gramsci). In interpersoneller Kommunikation zwischen antagonistischen Gruppen lassen sich diese Probleme - anders als Dewey impliziert - nicht lösen; vielmehr bilden sich mittlere Teilöffentlichkeiten und antagonistische Medien, die Public Relations betreiben, heraus (was der klassen-korporatistischen Ebene bei Gramsci entspricht). Finanzielle Ressourcen und soziales Kapital, wie etwa Beziehungsnetzwerke, sind zwischen den antagonistischen Medien ungleich verteilt, denn diese drücken unterschiedliche soziale Machtpositionen in der Gesellschaftsstruktur aus (z. B. arbeitgebernahe Wirtschaftsinstitute vs. Think Thanks aus dem Umfeld einer heterodoxen Ökonomik). Die Rolle organischer Intellektueller übernehmen Wissenschaftler*innen, PR-Spezialist*innen sowie einzelne Journalist*innen, die sehr direkt im Sinne der antagonistischen Interessen, z. B. als Kommentator*innen bereits auf der nächsten, komplexen Ebene der Massenmedien wirken.

Öffentliche Selbstvergesellschaftungs- und Problemlösungskompetenzen der Menschen werden im Sinne einer Kompromissbildung an die sich über der Gesellschaft konstitutierenden (Massen-)Medien abgegeben. Komplexe Öffentlichkeiten werden maßgeblich von ideologischen Mächten der Information und Kommunikation organisiert, was der hegemonial-politischen Ebene bei Gramsci entspricht. Die Massenmedien oder die ideologischen Apparate der Information und der Kommunikation sind verdichtete materialisierte Kräfteverhältnisse, in denen Kompromissbildungen ungleicher Interessen, die von den antagonistischen Medien verbreitet werden, materialisiert sind. Einige Interessen sind dabei gar nicht repräsentiert, wie z. B. wirtschaftspolitische Stimmen aus dem Umfeld heterodoxer Ökonomik, die kapitalistisches Wachstum in Frage stellen. Andere Stimmen, wie z. B. Arbeitnehmer*innen-nahe wirtschaftspolitische Positionen sind repräsentiert, aber innerhalb des ideologischen Rahmens des unbedingten Wirtschaftswachstums, den Arbeitgeber*innen-nahen Positionen untergeordnet. Ohne Wachstum, d. h. Profiten für 
Kapitalist*innen, können Arbeiter*innen-Interessen z. B. durch steuerliche Umverteilung nicht befriedigt werden. Das Ideologische trägt also zur relativen (aber restriktiven) Handlungsfähigkeit bei. Professionsregeln (z. B. der Ausgewogenheit und Unparteilichkeit) und ein journalistischer Habitus unterscheiden die Akteure in den Apparaten der Medien und der Kommunikation von den organischen Journalist*innen und Publizist*innen, Aktivist*innen und PR-Agent*innen (vgl. hierzu Bourdieu 2015).

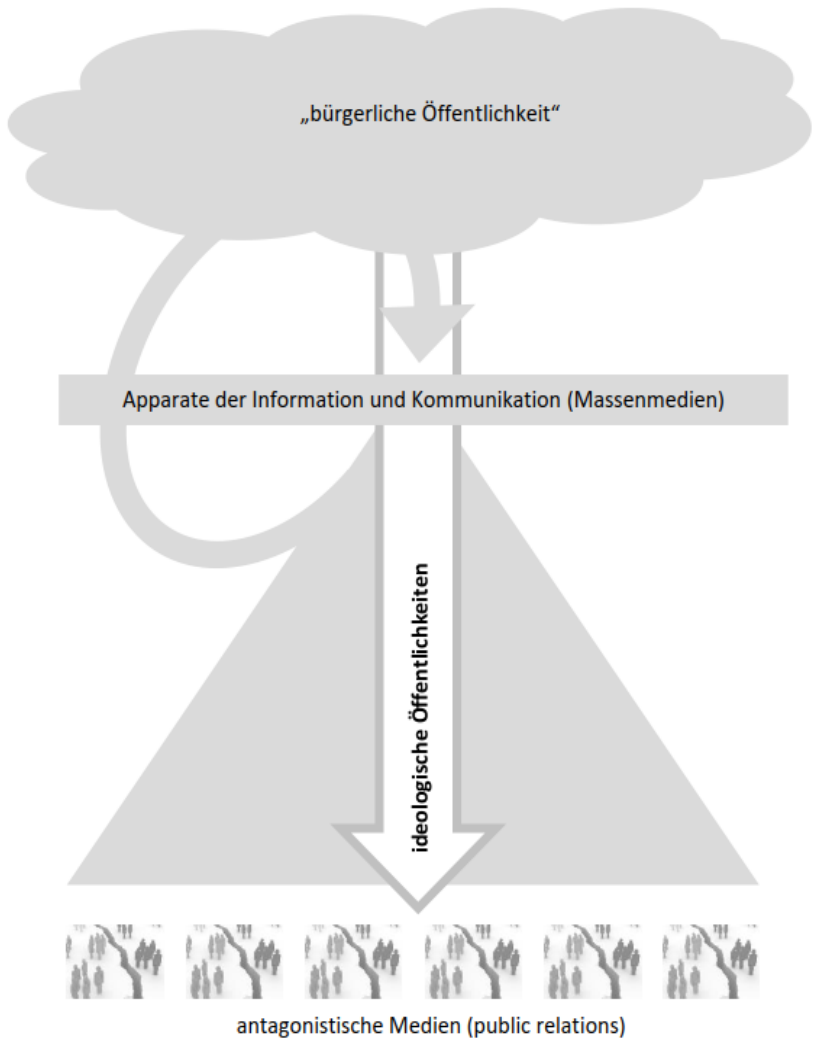

Öffentlichkeiten

Rekonstruktion des gesellschaftlichen Zusammenhang

Abb. 3: Wirkweisen ideologischer Öffentlichkeiten (eigene Darstellung) 
Die Massenmedien und die durch sie organisierten komplexen Öffentlichkeiten erheben den Anspruch, im Auftrag der "himmlischen Welt" zu handeln und das Gemeinwesen zu repräsentieren. Zur ideologischen Öffentlichkeit gehören neben den Massenmedien auch kompromissbildende Werte und Begriffe, wie z. B. Unparteilichkeit, herrschaftsfreier Diskurs, Presseethiken etc., über die sich die Massenmedien legitimieren und die sie verwalten, die aber auch an ihnen vorbei angerufen werden können.

Die "himmlische Welt" ist eine tendenziell universale, ausgewogene Öffentlichkeit, die das im Kapitalismus real zersplitterte Gemeinwesen ideell stiften soll. Mein Vorschlag ist es, den Begriff der „bürgerlichen Öffentlichkeit" (Habermas 1990; Negt und Kluge 1972) genau zur Charakterisierung dieser "himmlischen Welt" der Kommunikation zu verwenden. Gegen Habermas wurde von Negt und Kluge deutlich gemacht, dass die Idee bürgerlicher Öffentlichkeit selbst so sehr ein hegemoniales Klassenprojekt gewesen ist, um (koloniale) Ausbeutungsbeziehungen des entstehenden Bürgertums abzusichern (vgl. Negt und Kluge 1972, 33, 102-106), dass sie sich nicht eignet, um als Ideal den empirisch vorfindbaren Öffentlichkeiten kritisch gegenübergestellt zu werden. Bürgerliche Öffentlichkeit zeichnet sich dadurch aus, dass in ihr "der Hauptkampf gegen alle Besonderheiten geführt werden muß" (Negt und Kluge 1972, 31). In ihr wird von allen sozio-ökonomischen Unterschieden der Menschen abstrahiert, ausgebeutete Arbeiter*innen wie ausbeutende Kapitalist*innen kommen hier gleichermaßen als freie und gleiche Privatpersonen vor. Diese Einebnung von wirklichen Unterschieden hat zur Folge, dass sich unmittelbar aus den gemachten Erfahrungen ergebende politische Impulse, die auf den unteren Ebenen der Öffentlichkeit noch organisierend wirken, hier keinen Raum bekommen. Das „Über-alle-Situationen-hinweg-abstrakt-Reden“ (Negt und Kluge 1972, 91) wird vom Handeln, das durch Erfahrungen informiert ist, abgetrennt. Bürgerliche Öffentlichkeit zwingt die Erfahrungen in die Abstraktheit und suggeriert Allgemeinheit, wo diese in der gesellschaftlichen Wirklichkeit fehlt. Dadurch wirkt sie konservierend zu Gunsten derer, die vom Status quo (überproportional) profitieren.

Ideologische Apparate der Medien und der Kommunikation bzw. die Massenmedien repräsentieren bürgerliche Öffentlichkeit in unterschiedlichem Maße. Der öffentlich-rechtliche Rundfunk in seinen Hauptformaten, wie etwa den reichweitenstarken Meinungs- und Informations- 
sendungen, beansprucht z. B. in der BRD für sich, „, bürgerliche Öffentlichkeit" besser zu repräsentieren als dies z. B. Springer-Medien tun. Die bürgerliche Öffentlichkeit kann allerdings an ihren medialen Repräsentant*innen vorbei von unteren Ebenen der Öffentlichkeit bzw. antagonistischen Medien angerufen werden. Und zwar sowohl von den beherrschten Gruppen (im Sinne eines linken Populismus) als auch im Interesse der herrschaftlichen Absicherung antagonistischer Verhältnisse, die meint, zunehmend auf die in den Massenmedien verdichtete Kompromissbildung zwischen Interessen verzichten zu können (im Sinn eines autoritären Populismus). Populistische Momente ergeben sich immer dann, wenn Hegemonie im großen Stil neu verhandelt wird (vgl. z. B. Hall 2014a). Ziel der Populist*innen ist es jeweils, die Werte bürgerlicher Öffentlichkeit, also die kommunikative Repräsentation des Gemeinwesens, ohne Umweg über bestehende Kompromissbildung anzurufen und so im Falle einer hegemonialen Strategie neue Kompromissbildungen und Verdichtungen zu etablieren, wodurch gesellschaftliche Kräfteverhältnisse verschoben werden'.

Darüber hinaus sollte aber nicht vergessen werden, dass öffentliche Hegemonie immer mit Zwang abgesichert wird. Hegemoniale Prozesse werden durch direkte und indirekte Formen der Zensur aus dem Block an der Macht in den exekutiven Organen des Staates z. B. mittels Verboten von Medien, Berufsverbote bzw. Entlassungen von Intellektuellen und Journalist*innen, staatliche Einflussnahme auf Veröffentlichungen, repressiver Druck auf den Quellenschutz usw. abgesichert.

So kann abschließend auch auf Grenzen der Theorie ideologischer Öffentlichkeit reflektiert werden. Ihr geht es nicht zuerst um den Ausschluss von Erfahrungen und Interessen aus der Öffentlichkeit und nur in einem sehr weiten Verständnis, um die Analyse „systematisch verzerrter Kommunikationsbedingungen" (vgl. Biskamp in diesem Band) - nämlich, wenn unter Kommunikationsbedingungen die gesamte gesellschaftliche Anlage des öffentlichen Kommunikationsprozess gemeint ist. Es geht vielmehr um ein Verständnis der (selektiven) Aufnahme von Interessen

$9 \quad$ vgl. zur Unterscheidung von populistischem und popularem Projekt Hall 2014b. Vor dem Hintergrund der Theorie des Ideologischen wäre es populistisch Kräfteverhältnisse im Rahmen der ideologischen Anordnung verschieben zu wollen; ein populares Projekt hingegen zielt darauf, die ideologische Konstellation zu verlassen, d. h. die Wirkrichtung der Organisierung von Erfolgt nachhaltig, von unten' und dies ist nur wenn gleichzeitig der antagonistische Charakter der Gesellschaft aufgehoben wird. 
und die ideologische Organisation von Erfahrung, die Zustimmung zu Herrschaftsverhältnissen bewirkt. Damit geht es ihr auch nicht zuerst um die Kritik von Propaganda, also Täuschungs- und Manipulationsstrategien (vgl. Zollmann in diesem Band) antagonistischer Medien oder der Massenmedien. Wenngleich diese als Strategien antagonistischer Medien eine wichtige Rolle für hegemoniale Verallgemeinerungsprozesse oder als Indikator einer Verschiebung von Konsens in Richtung Zwang auch in den Massenmedien, etwa in Zeiten des Umbruchs spielen können.

Tab. 1: Ideologietheoretische Begriffe und ihre öffentlichkeitstheoretische Anwendung

\begin{tabular}{|l|l|}
\hline Ideologietheoretische Begriffe & Öffentlichkeitstheoretische Anwendung \\
\hline Hegemonie & Öffentliche Meinung \\
\hline Alltagsverstand & Erfahrung \\
\hline Gesellschaftliche Gruppen und Klassen & Antagonistische Medien \\
\hline Intellektuelle & Journalist*innen und Publizist*innen \\
\hline Organische Intellektuelle & Journalist*innen und PR-Agent*innen \\
\hline Traditionelle Intellektuelle & $\begin{array}{l}\text { Etablierte Journalist*innen und Heraus- } \\
\text { geber*innen, Chefredakteure, Inten- } \\
\text { dant*innen }\end{array}$ \\
\hline Ideologische Werte & Bürgerliche Öffentlichkeit \\
\hline Ideologische Apparate & $\begin{array}{l}\text { Massenmedien (Ideologische Apparate } \\
\text { der Medien und der Kommunikation) }\end{array}$ \\
\hline Vergesellschaftungskompetenzen & $\begin{array}{l}\text { Informationelle Bedürfnisse und kom- } \\
\text { munikative Problemlösungskompeten- } \\
\text { zen }\end{array}$ \\
\hline Horizontale Selbstvergesellschaftung & $\begin{array}{l}\text { Emanzipatorische Öffentlichkeiten: Re- } \\
\text { konstruktion des gesellschaftlichen Zu- } \\
\text { sammenhangs; Gewinn von Handlungs- } \\
\text { fähigkeit in verallgemeinerter Perspek- } \\
\text { tive; Erfahrungswachstum }\end{array}$ \\
\hline
\end{tabular}

Die Theorie ideologischer Öffentlichkeiten verhält sich komplementär und kompatibel zu diesen wichtigen Kritiken von Öffentlichkeit. Für Gramsci ist Hegemonie immer "gepanzert mit Zwang" (Gramsci 1991ff., 783), d. h. hegemoniale Mechanismen mischen sich real mit andersartigen Formen von Herrschaft, die mehr auf Zwang, Dominanz, Täuschung und Manipulation beruhen. Die Theorie ideologischer Öffentlichkeit ist selbst $\mathrm{zu}$ historisieren, es ist zu reflektieren, welche und wie große Teile der Bevölkerung hegemonial, also mittels Zustimmung herrschaftlich integriert 
werden. Die selektive und hierarchisierende Aufnahme von Erfahrungen ist begleitet von Ausschluss von Öffentlichkeit und Nicht-Repräsentanz für andere. Zudem sind „mögliche Ungleichzeitigkeiten zwischen kulturellen, politischen und ökonomischen Momenten der Hegemonie" (Martin und Wissel 2015, 224) zu berücksichtigen. Dies kann z. B. bedeuten, dass der Bereich öffentlicher (Massen-)Kommunikation noch weitgehend hegemonial funktioniert, während in den politischen und ökonomischen Bereichen und ihren (defizitären) Öffentlichkeiten Herrschaft mit weit weniger Zustimmung und Konsens etabliert wird. Aber auch die ideologischen Apparate der Medien und der Kommunikation haben politische und ökonomische Voraussetzungen, die mehr oder weniger hegemonial organisiert sein können. Auch hier, gleichsam aus der Innenperspektive einer Krise des Mediensystems betrachtet, können sich Ungleichzeitigkeiten einstellen und z. B. wenig konsensuelle Ausbildungs-, Rekrutierungsund Organisationsstrukturen der Massenmedien mit dem außenpluralistischen Anspruch breiterer Teile der Bevölkerung in Widerspruch geraten, in der Berichterstattung repräsentiert zu werden.

\section{8 Öffentlichkeiten im Vergesellschaftungsprozess}

Die Theorie der ideologischen Öffentlichkeiten, die hier in Umrissen dargelegt worden ist, gewinnt ihren kritischen Stachel vor dem Hintergrund der Selbstvergesellschaftungskompetenzen der Menschen als Gegenpart zur ideologischen Fremdvergesellschaftung. Was ist darunter aus öffentlichkeitstheoretischer Perspektive zu verstehen? Bevor diese Frage im abschließenden Abschnitt aufgegriffen werden kann, muss zunächst die generelle Notwendigkeit von Öffentlichkeit für Vergesellschaftungsprozesse, seien sie heteronom oder autonom, dargelegt werden.

Die Bildung von und Teilnahme an Öffentlichkeiten beruht auf der motivationalen Basis informationeller Bedürfnisse, die sich in der Menschwerdung herausbilden (vgl. Sevignani 2019): Anders als etwa einfache Organismen sind Menschen mit dem Entstehen von Gesellschaften nur noch mittelbar mit ihren Reproduktionserfordernissen verbunden. Es entstehen individuelle Freiräume, die die Voraussetzungen für eine handlungsfähige, reflexive, bewusste Subjektivität sind. Subjekte befinden sich in einer Möglichkeitsbeziehung zur Welt und können sich zu den gesellschaftlichen Bedingungen verhalten. Zwischen Bedingung und Handeln 
schiebt sich das subjektive Bedeuten und Begründen. Zu den Bedingungen des Handelns gehören auch gesellschaftliche Bedeutungsstrukturen und die wiederum können ideologische Angebote der Kompetenzaufgabe und Kompromissbildung sein. Es geht für eine so konturierte subjektwissenschaftlich fundierte Öffentlichkeitstheorie um die Aufklärung je spezifischer Bedingungs-Bedeutungs-Prämissen-Gründe-Zusammenhänge. Nur so ist zu erfahren, warum es subjektiv funktional ist, Kompetenzen an ideologische Instanzen abzugeben. Dabei sind Gründe immer aus der Perspektive erster Person zu verstehen. Sie können nur vom Standpunkt des Subjekts formuliert werden (je meine Gründe, in je meiner Situation). Wenn von außen behauptet wird, jemand andere handle irrational, dann hat derjenige, der das Verdikt äußert, sich nicht die Mühe gemacht, mit dem Betroffenen zu sprechen und dessen PrämissenGründe-Zusammenhang nachzuvollziehen; das heißt allerdings nicht, dass das Gegenüber den nachvollzogenen Zusammenhang teilen und übernehmen muss (vgl. Markard 2000).

Öffentlichkeiten werden erstmals nötig als Orte, wo der gesellschaftliche Zusammenhang und damit die eigene Position im gesellschaftlichen Gefüge rekonstruiert werden muss und für die Individuen nicht mehr unmittelbar einsichtig ist. In der Öffentlichkeit wird aber kein direkter Zugang zum Verstehen gesellschaftlicher Bedingungen eröffnet. Vielmehr treffen Subjekte mit unterschiedlichen Begründungen eigenen Handelns unter je unterschiedlich wahrgenommenen, aber dennoch objektiv gegebenen Bedingungen aufeinander. In Öffentlichkeiten kann ausgehend von den Begründungen der Subjekte, so oder so zu handeln, über die Explikation ihrer Prämissen (also den Bedeutungen der Bedingungen, die jeweils für das eigene Handeln aktualisiert werden) Verständigung im Sinne eines "metasubjektiven Standpunkt" (vgl. Holzkamp 1996) erreicht werden. Ein solcher Standpunkt ist perspektivisch möglich, weil die Menschen voneinander abhängen und unter verbindenden gesellschaftlichen Verhältnissen leben. Die subjektiven Möglichkeitsräume stehen nicht beziehungslos nebeneinander, sondern sind unterschiedliche Ausschnitte gesellschaftlicher Handlungsmöglichkeiten. Die Möglichkeitsverallgemeinerung besteht nun darin, die eigenen Handlungsmöglichkeiten als Fall von typischen Handlungsmöglichkeiten zu erkennen. Also Gemeinsamkeiten in den Möglichkeiten zu erkennen, die die Gesellschaft etwa für Frauen, Migrant*innen, und Lohnarbeiter*innen bietet oder ihnen verwehrt. 
Diese Notwendigkeit aber, auch die motivationale Grundlage für Öffentlichkeiten, die sich aus dem modernen Vergesellschaftungsprozess generell ergeben, verschärfen sich noch einmal in modernen kapitalistischen Gesellschaften. Denn diese zeichnen sich durch eine „blinde“ und unbewusste Vergesellschaftung über den Warentausch aus. Öffentlichkeit ist hier „,die einzige Ausdrucksform, welche die über den Produktionsprozeß lediglich ,privat' zusammengefügten Gesellschaftsglieder durch Zusammenfassung ihrer entfalteten gesellschaftlichen Eigenschaften miteinander verbindet" (Negt und Kluge 1972, 19).

\section{Emanzipatorische Öffentlichkeiten und die Perspektive horizontaler Selbstvergesellschaftung}

Nachdem die generelle Notwendigkeit und Möglichkeit von Öffentlichkeiten in modernen kapitalistischen Gesellschaften skizziert wurde, stellen sich für die kritische Kommunikationsforschung folgende Anschlussfragen: Welche Rolle können Öffentlichkeiten nun bei der horizontalen Selbstvergesellschaftung unter kapitalistischen Bedingungen spielen? Was wären emanzipative Öffentlichkeiten aus Sicht einer kritischen Theorie? Aus der Theorie ideologischer Öffentlichkeit ergibt sich neben der vagen normativen Zielvorstellung horizontaler Selbstvergesellschaftung zunächst nur negativ, dass Öffentlichkeiten nicht ein Gemeinwesen ersetzen sollen, wo es aufgrund ungleicher antagonistischer Produktions- und Reproduktionsverhältnisse (noch) keines gibt. Darin liegt, wie gezeigt, die zentrale ideologische und Herrschaft stabilisierende Funktion von Öffentlichkeit.

Stattdessen, so meine These, müssten Öffentlichkeiten gesellschaftliche Erfahrungen so organisieren, dass sie eine Selbstverständigung über die eigene Einbezogenheit in gesellschaftliche Herrschaftsverhältnisse ermöglichen. Dies wäre der Ausgangspunkt für die Bildung emanzipatorischer, nicht-ideologischer Öffentlichkeiten. Dies soll nun mit Hilfe der bereits angewandten Theorietraditionen umrissen werden.

In subjektwissenschaftlicher Perspektive sind Subjekte in antagonistisch-kapitalistischen Verhältnissen prinzipiell vor eine konfliktreiche Abwägung gestellt, ob sie mit ihrem Handeln 
- bestehende Verhältnisse akzeptieren, sich also mit der antagonistischen Situation arrangieren,

- ihre Handlungsfähigkeit im Zusammenschluss mit anderen erweitern, dies aber auf Kosten anderer geschieht, oder

- die Bedingungen perspektivisch unter dem Einschluss aller anderen so verändern, dass Herrschaftsverhältnisse aufgehoben werden.

Die ersten beiden Möglichkeiten können restriktive Handlungsfähigkeit und die letzte Option verallgemeinerte Handlungsfähigkeit genannt werden (vgl. Holzkamp 1990; Meretz 2016). Restriktiv sind die ersten beiden Optionen, weil subjektive Handlungsfähigkeit grundsätzlich in einer arbeitsteiligen und darüber hinaus auf Ausbeutungsverhältnissen beruhenden kapitalistischen Gesellschaft immer von den Handlungen anderer abhängig ist. Basieren diese Abhängigkeiten aber auf Ungleichheit, wie im Fall der Ausbeutung, stehen sie jederzeit in Gefahr, herausgefordert zu werden. Die eigene Reproduktion, die auf andere angewiesen ist und herrschaftlich abgesichert wird, ist prekär, denn sie geht mit der Gefahr einher, dass sie von den Beherrschten Frage gestellt wird.10 Für Widerstand in diesem Sinne bildet, wie dargelegt, die subjektive Erfahrung den Ausgangspunkt. Welche der oben genannten Handlungsoptionen subjektiv vernünftig erscheint, kann nicht vom Standpunkt dritter Personen beurteilt werden. Die Optionen bilden also keine externen Kriterien zur Beurteilung von konkretem Handeln. Vielmehr ist der Subjektstandpunkt durch eine Verständigung über die Bedingungen, die zugrunde gelegten Prämissen und die jeweils subjektiven Gründe zu rekonstruieren, und die Prekarität eigener restriktiver Handlungsfähigkeit muss jeweils nachvollziehbar werden.

Dies setzt mindestens voraus, dass einmal die Widersprüchlichkeiten und Vielfältigkeit manifester Interessen unterschiedlicher Akteure in der Öffentlichkeit wahrgenommen werden und dass dann die Genese und Bedingungen dieser Interessen öffentlich rekonstruiert werden. Auf dieser Basis können im öffentlichen Diskurs Ideen und Haltungen entwickelt werden, die es erlauben, Widersprüche konstruktiv aufzuheben oder Interessen schlüssig zwischen den Akteuren zu priorisieren, aber dabei auch sich selbst, d. h. die eigenen möglicherweise widersprüchlichen 
Handlungsprämissen und -begründungen zu transformieren. Diese subjektwissenschaftliche Perspektive auf die Leistungen emanzipatorischer Öffentlichkeiten entspricht Kohärent-Arbeiten des widersprüchlichen und fragmentierten Alltagsverstandes nach Gramsci, das an seinem "gesunden Kern“, also der Unmittelbarkeit der Erfahrung (von unten) und den Teilen ansetzen muss, die der (ideologisch organisierten) öffentlichen Meinung widersprechen. Mit der subjektwissenschaftlichen Perspektive des notwendigen Nachvollzugs von unterschiedlichen Handlungsbegründungen wird aber die notwendige radikaldemokratische Organisation von Öffentlichkeit betont.

Gramsci unterscheidet zwischen der Funktion der Religion als "Opium des Volkes" (Marx) einerseits, die zwar den Alltagsverstand hört und versteht, aber ihn in seiner bizarren Form der Widersprüchlichkeit und Uneinheitlichkeit belässt, und der Philosophie (der Praxis) andererseits, die den Alltagsverstand kohärent machen kann und der an emanzipatorischen Öffentlichkeiten gelegen sein muss. Eine öffentlichkeitstheoretische Entsprechung zur Funktion der Religion findet sich bei den von Negt und Kluge als Zerfallsform bürgerlicher Öffentlichkeit analysierten Produktionsöffentlichkeiten: „Die traditionelle Öffentlichkeit, deren charakteristische Schwäche auf dem Ausgrenzungsmechanismus zwischen öffentlich und privat beruht, wird heute überlagert von industrialisierten Produktionsöffentlichkeiten, die die Tendenz haben, Privatbereiche, insbesondere den Produktionsprozeß und den Lebenszusammenhang, einzubeziehen" (Negt und Kluge 1972, 35). ${ }^{11}$ Massenwirksamkeit erreichen diese profitorientierten Akteure der Produktionsöffentlichkeit dadurch, dass sie von den realen Erfahrungen und Wünschen der Massen gerade nicht abstrahieren und sich auf Konkretes einlassen. Einerseits beeinflussen PR-Strategien einflussreicher Akteure, so die Zeitdiagnose, zunehmend ungebrochen durch Kompromissbildungen die Öffentlichkeit. Andererseits werden Erfahrungen auch der Ausgebeuteten und Unterdrückten unmittelbar aufgenommen, dabei aber, so Negt und Kluge, in ihrer Unmittelbarkeit und Privatheit festgehalten. Dadurch ist eine aus emanzipatorischer Perspektive notwendige Ausarbeitung und Auseinander-

11 Zu diskutieren wäre, inwieweit Öffentlichkeiten, die sich in den Sozialen Medien konstituieren, die ja auch durch eine Vermischung von Privatem und Öffentlichem gekennzeichnet sind, eine Variante der Produktionsöffentlichkeiten darstellen (vgl. hierzu z. B. Dean 2014). 
setzung mit ähnlichen und konträren Erfahrungen verstellt. Das Ergebnis der sich überlappenden bürgerlichen und Produktionsöffentlichkeiten ist „ein Hin- und Herschwanken zwischen Ausgrenzung und verstärkter Einbeziehung“ (Negt und Kluge 1972, 38): „Der proletarische Lebenszusammenhang wird so in zwei Teile aufgespalten: Der eine wird in die neuen Produktionsöffentlichkeiten resorbiert, nimmt an der Industrialisierung teil, der andere wird im Sinne der die Gesellschaft bestimmenden Systeme von Produktion und Produktionsöffentlichkeit dequalifiziert" (Negt und Kluge 1972, 43).

Negt und Kluge verwenden letztlich einen emphatischen Erfahrungsbegriff in der Tradition der älteren Frankfurter Schule vor dessen Hintergrund die Blockierung von „echten“ Erfahrungen, die auf den Zusammenhang von Erfahrungen zielt, zum Problem wird. Erfahrung kann zwar durch gesellschaftliche Institutionen wie die Massenmedien blockiert werden, aber in den Erfahrungen, wie im Verständnis des Alltagsverstandes nach Gramsci, lässt sich gleichzeitig auch etwas Widerständiges ausmachen, das gegen eine Blockierung gerichtet werden kann. Negt und Kluge sprechen in diesem Zusammenhang von den Erfahrungen der "lebendigen Arbeit", wie etwa Fantasie, Solidaritätsbedürfnis, Orientierungsbedürfnis, sowie einem materialistischen Instinkt, der durch Einschränkungen von Lebensmöglichkeiten und Handlungsfähigkeit geweckt wird. Ähnlich wie der gesunde Kern des Alltagsverstandes bei Gramsci lassen sich Bedürfnisse und Wünsche nicht völlig in kapitalistische Verhältnisse einpassen; sie bilden den Erfahrungsrohstoff der auch anders - mittels proletarischer Öffentlichkeit organisiert - werden kann und dann nicht unterdrückt oder regressiv umgeformt würde (vgl. Negt und Kluge 1972, 67-83). Blockierung und Dequalifizierung von Erfahrung findet dann statt, wenn einzelne Erfahrungen isoliert bleiben und aus ihrem Zusammenhang gerissen werden oder aber der Erfahrungszusammenhang nur abstrakt gestiftet wird, d. h. konkrete Erfahrungen in ihm verschwunden sind (vgl. Negt und Kluge 1972, 60ff.). Demgegenüber setzen Negt und Kluge auf einen Lernprozess der "Erfahrung in der Herstellung von Erfahrung" (Negt und Kluge 1972, 27) bzw. der proletarischen Öffentlichkeit bezeichnen und der bürgerlichen Öffentlichkeit und den Produktionsöffentlichkeiten als antagonistisches Prinzip gegenüberstellen. Diese Öffentlichkeit ist nicht identisch mit empirischen Arbeiterinnen-Öffentlichkeiten, sondern in weiten "Strecken mit dem identisch, was in der marxistischen Tradition Klassenbewußtsein und Klassen- 
kampf heißt. Diese letzteren Begriffe bezeichnen jedoch Resultate, nicht die Vermittlung und die Entstehungsbedingungen und den konkreten Zusammenhang ihrer einzelnen Elemente“ (Negt und Kluge 1972, 66f.).

Mit Dewey kann eine weitere Konturierung des Prozesses der Erfahrung in der Herstellung von Erfahrungen bzw. emanzipatorischen Öffentlichkeiten vorgenommen werden: Für ihn können Problemlösungen und deren Erprobung entweder sehr zufällig und unkontrolliert oder aber nach Vorbild der experimentellen Wissenschaften methodisch kontrolliert und in Austausch und Kooperation mit anderen Betroffenen und deren organischen Intellektuellen (bei Dewey Wissenschaftler*innen) erfolgen. Diese experimentalistische Form der Problemlösung ist für Dewey die präferierte Option, weil sie effizientere Lösungen für die Betroffenen verspricht und nicht nur erneute Anpassung an die Situation garantiert, sondern auch ein Wachstum von Erfahrungen ermöglicht. Mit Dewey kann also einerseits an die skizzierten kritischen Theorien angeknüpft werden: Erfahrungswachstum bei Dewey bedeutet verallgemeinerte Handlungsfähigkeit in subjektwissenschaftlicher Perspektive, KohärentArbeiten des Alltagsverstands aus hegemonietheoretischer Perspektive und die Erfahrung im Herstellen von Erfahrung aus der Perspektive der Theorie proletarischer Öffentlichkeit. Zugleich ergänzt er diese aber um ein epistemologisches Argument, wonach emanzipatorische Öffentlichkeiten effizientere Problemlösungen ermöglichen (vgl. Honneth 2000, 293).

Zusammenfassend haben emanzipatorische Öffentlichkeiten also folgende Merkmale, vor deren Hintergrund die Theorie ideologischer Öffentlichkeiten ihren (kapitalismus-)kritischen Stachel erhält:

- Statt ein Gemeinwesen zu simulieren, geht es in emanzipatorischen Öffentlichkeiten um die Wahrnehmung bzw. Repräsentation antagonistischer gesellschaftlicher Interessen mittels einer Rekonstruktion ihrer Genese und Bedingungen.

- Diese Rekonstruktion schließt auch ein, dass die eigene Verstrickung in gesellschaftliche Herrschaftsverhältnisse im Austausch mit anderen rekonstruiert wird.

- Ansatzpunkt ist die Unmittelbarkeit der Erfahrung (von unten) und den damit verbundenen Intuitionen, Gefühlen und Gedanken, die der öffentlichen Meinung widersprechen. 
- Und zwar mit dem Ziel, diese Erfahrung so zu organisieren, dass der widersprüchliche und fragmentierte Alltagsverstand kohärenter wird.

- Kohärenz lässt sich in der Perspektive verallgemeinerter Handlungsfähigkeit gewinnen, die nur durch den Zusammenschluss mit anderen erreicht werden kann.

- Kooperiert wird öffentlich, um die Bedingungen, die zu den Problemerfahrungen führten, möglichst effizient im Sinne der Betroffenen zu verändern.

Damit sind einige Eckpunkte emanzipatorischer bzw. anti-ideologischer Öffentlichkeit benannt, die etwa mit Bezug auf die Erfahrungen realer Klassenbildung (z. B. Thompson 1987) und der Formierung (neuer) sozialer Bewegungen (z. B. Stamm 1998) - auch im Hinblick auf digitale Kommunikation und soziale Medien vertiefend, ergänzend oder korrigierend diskutiert werden müssen.

\section{Literatur}

Becker, Lia, Mario Candeias, Janek Niggemann, und Anne Steckner, Hrsg. 2013. Gramsci lesen: Einstiege in die Gefängnishefte. Hamburg: Argument.

Boltanski, Luc, und Ève Chiapello. 2006. Der neue Geist des Kapitalismus. 1. Aufl. Konstanz: UVK.

Bourdieu, Pierre. 2015. Über das Fernsehen. Berlin: Suhrkamp.

Dean, Jodi. 2014. „Communicative Capitalism and Class Struggle“. Spheres: Journal for Digital Cultures 1: 1-16.

Demirović, Alex. 1997. Herrschaft und Demokratie: Aspekte kritischer Gesellschaftstheorie. Münster: Westfälisches Dampfboot.

Demirović, Alex. 1999. Der nonkonformistische Intellektuelle: Die Entwicklung der Kritischen Theorie zur Frankfurter Schule. Frankfurt am Main: Suhrkamp.

Demirović, Alex. 2018. „Das Geschlechterverhältnis und der Kapitalismus: Plädoyer für ein klassenpolitisches Verständnis des multiplen Herrschaftszusammenhang". In Kapitalismuskritische Gesellschaftsanalyse: queer-feministische Positionen, herausgegeben von Katharina Pühl und Birgit Sauer, 258-85. Münster: Westfälisches Dampfboot.

Demirović, Alex, und Peter Jehle. 2005. „Intellektuelle“. In Historisch-Kritisches Wörterbuch des Marxismus (HKWM). Bd. 6/2. Berlin: Argument.

Dewey, John. 1986. „Kriterien der Erfahrung“. In John Dewey: Erziehung durch und für Erfahrung, herausgegeben von Helmut Schreier, 283-96. Stuttgart: Klett-Cotta.

Dewey, John. 1996. Die Öffentlichkeit und ihre Probleme. Bodenheim: Philo. 
Downey, John, Gavan Titley, und Jason Toynbee. 2014. „Ideology critique: the challenge for media studies". Media, Culture $\mathcal{E}$ Society $36 \quad$ (6): $878-87$. https://doi.org/10.1177/0163443714536113.

Eagleton, Terry. 2000. Ideologie: Eine Einführung. Übersetzt von Anja Tippner. 1. Stuttgart: J.B. Metzler.

Fraser, Nancy. 2001. „Neue Überlegungen zur Öffentlichkeit: Ein Beitrag zur Kritik real existierender Demokratie". In Die halbierte Gereichtigkeit: Schlüsselbegriffe des postindustriellen Sozialstaats, 107-50. Frankfurt am Main: Suhrkamp.

Fraser, Nancy, und Rahel Jaeggi. 2018. Capitalism: A Conversation in Critical Theory. 1. Cambridge: Polity.

Götz, Annika. 2017. Kritik der Öffentlichkeiten: John Dewey neu denken. Berlin: Springer.

Gramsci, Antonio. 1991ff.. Gefängnishefte. Herausgegeben von Klaus Bochmann und Wolfgang Fritz Haug. Hamburg: Argument.

Habermas, Jürgen. 1990. Strukturwandel der Öffentlichkeit: Untersuchungen zu einer Kategorie der bürgerlichen Gesellschaft. Frankfurt am Main: Suhrkamp.

Hall, Stuart. 1989. „Der Thatcherismus und die Theoretiker“. In Ausgewählte Schriften: Ideologie, Kultur, Medien, Neue Rechte, Rassismus, herausgegeben von Nora Räthzel, 172206. Berlin: Argument.

Hall, Stuart. 1994. „,'Rasse', Artikulation und Gesellschaft mit struktureller Dominante“. In Rassismus und kulturelle Identität: Ausgewählte Schriften 2, herausgegeben von Nora Räthzel, 89-136. Hamburg: Argument.

Hall, Stuart. 2014a. „Die Bedeutung des autoritären Populismus für den Thatcherismus“. In Populismus, Hegemonie, Globalisierung: Ausgewählte Schriften 5, herausgegeben von Juha Koivisto, Ingo Lauggas, und Victor Rego Diaz, 121-32. Hamburg: Argument.

Hall, Stuart. 2014b. „Eine permanente neoliberale Revolution?" In Populismus, Hegemonie, Globalisierung: Ausgewählte Schriften 5, herausgegeben von Juha Koivisto, Ingo Lauggas, und Victor Rego Diaz, 228-53. Hamburg: Argument.

Haug, Wolfgang Fritz. 1984. „Die Camera obscura des Bewußtseins: Kritik der Subjekt/Objekt-Artikulation im Marxismus". In Die Camera obscura der Ideologie: Philosophie - Ökonomie - Wissenschaf, herausgegeben von Projekt Ideologie-Theorie (PIT), 9-96. Berlin: Argument.

Haug, Wolfgang Fritz. 1993. Elemente einer Theorie des Ideologischen. Berlin: Argument.

Holzkamp, Klaus. 1990. „Worauf bezieht sich das Begriffspaar, restriktive/ verallgemeinerte Handlungsfähigkeit'? Zu Maretzkys vorstehenden ,Anmerkungen'“. Forum Kritische Psychologie 26: 35-45.

Holzkamp, Klaus. 1996. „Psychologie: Selbstverständigung über Handlungsbegründungen alltäglicher Lebensführung". Forum Kritische Psychologie 36: 7-112.

Honneth, Axel. 2000. „Demokratie als reflexive Kooperation. John Dewey und die Demokratietheorie der Gegenwart". In Das Andere der Gerechtigkeit: Aufsätze zur praktischen Philosophie, 282-309. Frankfurt am Main: Suhrkamp.

Jörke, Dirk. 2003. Demokratie als Erfahrung: John Dewey und die politische Philosophie der Gegenwart. VS Verlag für Sozialwissenschaften. 
Jörke, Dirk. 2007. „John Dewey über Erfahrung, Demokratie und Erziehung“. In Demokratiebewusstsein: Interdisziplinäre Annäherungen an ein zentrales Thema der Politischen Bildung, herausgegeben von Dirk Lange und Gerhard Himmelmann, 2007. Aufl., 87-98. Wiesbaden: VS Verlag für Sozialwissenschaften.

Klaus, Elisabeth, und Ricarda Drüeke. 2017. Öffentlichkeiten und gesellschaftliche Aushandlungsprozesse, Theoretische Perspektiven und empirische Befunde. 1. Aufl. Bielefeld: transcript Verlag. https:// doi.org/10.14361/9783839430491.

Klaus, Elisabeth, und Ulla Wischermann. 2008. „Öffentlichkeit als Mehr-Ebenen-Prozess Theoretische Uberlegungen und empirische Befunde am Beispiel der Frauenbewegungen um 1900". Zeitschrift für Frauenforschung \& Geschlechterstudien 26 (3/4): 103-16.

Koivisto, Juha, und Veikko Pietilä. 1996. „Ideological powers and resistance: The contribution of W. F. Haug and Projekt Ideologie-Theorie“. Rethinking Marxism 9 (4): 40-59.

Laclau, Ernesto. 2010. „Was haben leere Signifikanten mit Politik zu tun?“ In Emanzipation und Differenz, übersetzt von Oliver Marchart, 65-78. Wien Berlin: Turia + Kant.

Marchart, Oliver, Hrsg. 2017. Ordnungen Des Politischen: Einsätze Und Wirkungen Der Hegemonietheorie Ernesto Laclaus. Staat - Souveränität - Nation. Wiesbaden: VS Verlag für Sozialwissenschaften.

Markard, Morus. 2000. „Kritische Psychologie: Methodik vom Standpunkt des Subjekts“. Forum Qualitative Sozialforschung / Forum: Qualitative Social Research 1 (2). http://www.qualitative-research.net/index.php/fqs/article/view/1088. Zugegriffen: 15. November 2019.

Martin, Dirk, und Jens Wissel. 2015. „Fragmentierte Hegemonie: Anmerkungen zur gegenwärtigen Konstellation von Herrschaft". In Konstellationen und Perspektiven kritischer Theorien, herausgegeben von Dirk Martin, Susanne Martin, und Jens Wissel, 220-39. Münster: Westfälisches Dampfboot.

Meretz, Stefan, und Denis Neumüller. 2016. „Erweiterung oder Verallge-meinerung der Handlungs-fähigkeit?“ Berlin. http://2016.ferienuni.de/files/denis-neumueller-stefan-meretz-verallgemeinerte-handlungsfaehigkeit.pdf. Zugegriffen: 15. November 2019.

Negt, Oskar, und Alexander Kluge. 1972. Öffentlichkeit und Erfahrung. Zur Organisationsanalyse von bürgerlicher und proletarischer Öffentlichkeit. Frankfurt am Main: Suhrkamp.

Opratko, Benjamin. 2014. Hegemonie: Politische Theorie nach Antonio Gramsci. 2. Aufl. Münster: Westfälisches Dampfboot.

Projekt Ideologie-Theorie (PIT). 1979. Theorien über Ideologie. Berlin: Argument.

Rehmann, Jan. 2008. Einführung in die Ideologietheorie. 4. Aufl. Hamburg: Argument.

Reitz, Tilman. 2004. "Ideologiekritik“. In Historisch-Kritisches Wörterbuch des Marxismus (HKWM), 6.1:690-717. Berlin: Argument.

Ritsert, Jürgen. 2002. Ideologie: Theoreme und Probleme der Wissenssoziologie. 2. Aufl. Münster: Westfälisches Dampfboot.

Ritzi, Claudia. 2013. Die Postdemokratisierung politischer Öffentlichkeit: Kritik zeitgenössischer Demokratie - theoretische Grundlagen und analytische Perspektiven. Springer. 
Sevignani, Sebastian. 2016. „Kritische Politische Ökonomie“. In Handbuch Medienökonomie, herausgegeben von Jan Krone und Tassilo Pellegrini, 1-18. Wiesbaden: Springer Fachmedien Wiesbaden. https:/ / doi.org/10.1007/978-3-658-09632-8_3-1

Sevignani, Sebastian. 2018. „Historisch-Materialistische Medien- und Kommunikationstheorie 2.0". Maske und Kothurn: Internationale Beiträge zur Theater-, Film- und Medienwissenschaft 64 (1/2): 59-88.

Sevignani, Sebastian. 2019. "The development of informational needs and prospects of a need-based critique of digital capitalism“. Annual Review of Critical Psychology 14.

Stamm, Karl-Heinz. 1998. Alternative Öffentlichkeit: Die Erfahrungsproduktion neuer sozialer Bewegungen. Frankfurt am Main: Campus.

Thompson, Edward P. 1987. Die Entstehung der englischen Arbeiterklasse, 2 Bde. Frankfurt am Main: Suhrkamp.

Žižek, Slavoj, Hrsg. 1995. Mapping Ideology. London: Verso.

\section{Open Access}

Dieser Beitrag erscheint unter der Creative-Commons-Lizenz CC-BY-SA 4.0:

https://creativecommons.org/licenses/by-sa/4.0/ 